\title{
Analysis of Survival of Patients with Brain Metastases According to Prognostic Indexes and Treatment Strategies
}

\author{
Halil SAGINC ${ }^{1}$, P. Bahar BALTALARLI², Ergin SAGTAS ${ }^{3}$, M. Erdal COSKUN ${ }^{4}$ \\ ${ }^{1}$ Denizli State Hospital, Department of Radiation Oncology, Denizli, Turkey \\ ${ }^{2}$ Pamukkale University School of Medicine, Department of Radiation Oncology, Denizli, Turkey \\ ${ }^{3}$ Pamukkale University School of Medicine, Department of Radiology, Denizli, Turkey \\ ${ }^{4}$ Pamukkale University School of Medicine, Department of Neurosurgery, Denizli, Turkey \\ Corresponding author: Halil SAGINC halilsaginc@hotmail.com
}

\section{ABSTRACT}

AIM: To retrospectively evaluate the overall survival (OS) of patients with brain metastases (BMs) who had been treated with whole brain radiotherapy (WBRT) and Gamma Knife (GK) according to prognostic factors and prognostic index scores.

MATERIAL and METHODS: The study included 91 patients with BMs who had been treated with WBRT and/or GK between 2014 and 2017. The patients with BMs were retrospectively evaluated regarding age, sex, Karnofsky Performance Status (KPS), recursive partitioning analysis (RPA) class, basic score for BM (BS-BM), Graded Prognostic Assessment (DS-GPA) index, primary tumour type, extracranial metastases, primary tumour control, number of BMs, and brain metastasectomy. A univariate analysis of the OS was performed using the Kaplan-Meier method, supplemented by the log-rank test. We also applied a multivariate survival analysis using the Cox regression model.

RESULTS: The median OS for all patients with BMs was 6 months. Meanwhile, the median OSs for those with WBRT, GK, and WBRT-GK treatment were 6,4 , and 15 months, respectively $(p=0.00)$. In the multivariate analysis, the female sex $(p=0.030)$, brain metastasectomy $(p=0.047)$, treatment with WBRT-GK $(p=0.001)$, and the controlled primary tumour $(p=0.007)$ significantly correlated with the OS. Furthermore, the BS-BM ( $p=0.022)$ was closely related with the OS compared to the RPA and DS-GPA in the multivariate analysis.

CONCLUSION: The BS-BM was found to better predict the survival of patients with BMs according to the prognostic index scores in the multivariate analysis. Thus, our data suggest that the BS-BM is the most appropriate prognostic index.

KEYWORDS: Brain metastases, Survival, Recursive partitioning analysis (RPA), Basic score for brain metastases (BS-BM), Graded prognostic assessment (GPA) index

\section{INTRODUCTION}

$\mathrm{T}$ There is an increasing number of patients with brain metastases (BMs) (22). BMs is the most common brain tumour that develops in approximately $10 \%-40 \%$ of adult patients with cancer (27). Furthermore, the most common sources of BMs include lung cancer, melanoma, and breast cancer (29). The aims of treatment include the palliation of neurological symptoms, maintenance of performance status, and local control of the metastatic disease (3). Whole brain radiotherapy (WBRT) is commonly used to improve neurological symptoms in patients with multiple BMs and to control the disease within the brain $(1,7,27,29,32)$. Stereotactic radiosurgery is a good choice for treating oligometastatic brain lesions $(1,18,24)$, while a metastasectomy is also a treatment option. However, selection of local treatment only or WBRT depends on the performance of the patient, number of BMs, and histology (40). WBRT is the primary modality in patients with multiple BMs (2). For patients with oligo BMs
Halil SAGINC (1): 0000-0002-3269-0267

P. Bahar BALTALARLI (1) : 0000-0001-5317-3851
Ergin SAGTAS (D) : 0000-0001-6723-6593

M. Erdal COSKUN (1) : 0000-0003-3606-1844 
treated with radiosurgery or a metastasectomy, the addition of WBRT to the treatment regimen has reduced intracranial recurrence and neurological mortality. The principal problem in patients with the addition of WBRT is neurocognitive function failure. However, the functional independence and mean survival have not been improved (19). Chang et al. reported that radiosurgery and WBRT decreased learning and memory function compared to radiosurgery alone (8). While Tsao et al. reported that radiosurgery and WBRT improved the control of local and remote BMs compared to radiosurgery alone (41). The benefit of gamma knife (GK) radiosurgery is the reduction of radiation to the surrounding normal brain parenchyma, which may thereby reduce neurological toxicities compared with WBRT $(3,34)$ or may improve local control when combined with WBRT (1).

The KPS score, primary lesion control, presence of extracranial metastases, presence of multiple metastases are important prognostic factors in the literature $(12,23,39,40)$. Surgical resection provides improved disease control for patients with single and resectable metastasis (30).

In recent years, several prognostic scoring systems based on independent prognostic factors have been developed to evaluate the pre-treatment variable contributions, with the aim of choosing the appropriate treatment for individual patients, and to guide future research. The most widely used indices are the recursive partitioning analysis (RPA), basic score for BM (BS-BM), and the graded prognostic assessment (DSGPA) index $(12,23,39)$. Table I shows the clinical parameters used for the prognostic indices (RPA, DS-GPA, and BS-BM).
In this paper, the retrospective examination of the patients with BMs who had been treated with WBRT, GK, and combined WBRT-GK in a single centre are reported. We tried to determine the appropriate prognostic indexes for all patients with BMs who had undergone GK, WBRT, and combined WBRT-GK.

\section{MATERIAL and METHODS}

\section{Study Design}

This study included 91 patients with BMs who had undergone total WBRT, GK radiosurgery, or combined WBRT-GK between 2014 and 2017. Of these patients, 63 had lung cancer, 14 had breast cancer, 5 had colorectal cancer, 8 had genitourinary cancer, and one had a melanoma.

\section{Data Collection}

The data of the 91 patients were retrospectively collected and evaluated regarding the clinical characteristics, including age, sex, Karnofsky Performance Status (KPS), RPA Class, BSBM, DS-GPA, primary tumour type, extracranial metastases, primary tumour control, number of BMs, total BMs volume and brain metastasectomy. Prognostic indices, such as the RPA class, BS-BM, and DS-GPA, were applied to patients with BMs. These criteria were chosen in accordance with previous studies which identified significant predictors of survival in patients with BMs $(12,36,39,40)$.

\section{Study procedures WBRT}

Patients with multiple BMs were chosen for treatment with WBRT. Patients were immobilised in a supine position with

Table I: Clinical Parameters Used for Prognostic Indexes (RPA, DS-GPA, and BS-BM)

\begin{tabular}{|c|c|c|c|}
\hline & \multicolumn{3}{|c|}{ RPA } \\
\hline Class 2 & All patients not in Class 1 or 3 & & \\
\hline \multirow[t]{2}{*}{ Class 3} & KPS <70 & & \\
\hline & \multicolumn{3}{|c|}{ DS-GPA } \\
\hline Age, year & $>60$ & $50-59$ & $<50$ \\
\hline KPS & $<70$ & $70-80$ & $90-100$ \\
\hline Number of BM & $>3$ & $2-3$ & 1 \\
\hline ECM & Yes & & No \\
\hline KPS & $50-70$ & $80-100$ & \\
\hline Control of primary tumor & No & Yes & \\
\hline ECM & Yes & No & \\
\hline
\end{tabular}

KPS: Karnofsky performance status, RPA: Recursive partitioning analysis, BS-BM: Basic score for brain metastases, DS-GPA: Diseases specificgraded prognostic assessment, BM: Brain metastases, ECM: Extracranial metastases. 
a thermoplastic mask. The brain was contoured as a clinical target volume (CTV) until the foramen magnum; this CTV was equal to the planning target volume (PTV). All BMs, optic nerves, brainstems, eyes, and lenses were contoured while patients were positioned with the mask. Planning the CT was mandatory with a slice thickness of $\leq 5 \mathrm{~mm}$. Mean while, WBRT was performed with 6-MV photons from a Siemens Artiste linear accelerator. The daily prescription dose is 2.5 and 3 Gy, prescribed as the ICRU reference point.

\section{Study procedures: Radiosurgery}

The patients with 1-3 BMs of $<3 \mathrm{~cm}$ were chosen for the gamma knife treatment. Patients were immobilised in a supine position with a stereotactic fixation system using an invasive frame. A planned CT scan with $\leq 2 \mathrm{~mm}$ thick contiguous slices (preferable CT slice thickness $=1 \mathrm{~mm}$ ) was later fused to a contrast-enhanced stereotactic MRI scan. BMs were contoured using a CTV to PTV margin. Radiosurgery was performed with Elekta Leksell GK machine, doses of which ranged from 15-25 Gy.

\section{Patient Follow-up, Salvage Therapy}

The metastatic brain lesions of the patients in this study were tracked by MRI. The overall survival (OS) of a patient was referenced from the day of diagnosis of the BM by MRI. Furthermore, intracerebral failure was diagnosed by MRI. The exact frequency and number of MRls following irradiation were unavailable because the anonymised database used did not include these data. In general, the follow-up schedule after therapy includes an MRI every 3 months, whereas, here, an MRI was performed only in the cases of new or progressive symptoms in most patients undergoing WBRT or GK. All patients with BMs were treated after being evaluated by the Neurosurgery and Radiation Oncology Departments. Salvage stereotactic radio-surgery (SRS) was an additional treatment option for recurrent BMs after failure of the WBRT (45). After SRS, salvage WBRT was added to reduce intracranial relapses and neurological deaths (38). Patients who underwent only GK treatment or only WBRT treatment accepted salvage treatment under GK, under the WBRT, or combined treatment (WBRT$\mathrm{GK})$, respectively. We accepted patients who underwent salvage treatment due to progression after WBRT treatment or SRS treatment in the combined WBRT-GK.

\section{Statistical Analysis}

A univariate analysis of the OS was performed using the Kaplan-Meier method supplemented by the log-rank test to determine the factors (16). We also applied a multivariate survival analysis using the Cox regression model. Only the factors exhibiting statistical significance in the univariate analysis were included in multivariate analysis which utilised the Cox proportional hazards regression tests. All tests were two-tailed, and a p-value of $<0.05$ was considered significant. The statistical analyses were reviewed by a medical statistician stuff in our medical faculty.

\section{RESULTS}

\section{Patients Characteristics}

Among the 91 patients in this study, 69 (75.8\%) were men and
$22(24.2 \%)$ were women. The mean follow-up duration was 10.56 months, with a median of 6 months (min: 1 month, max: 57 months). The median age was 59 years (min: 31 years, max: 83 years). The number of patients who underwent WBRT, GK radiosurgery, and combined WBRT-GK was 43 (47.3\%), $23(25.3 \%)$, and $25(27.5 \%)$, respectively. In our study, 38 patients with only WBRT treatment, 18 patients with only GK radiosurgery treatment, and 17 patients with combined WBRTGK treatment died. The median OS for all patients with BMs was 6 months (range: 1-57 months; 95\% Cl: 3.664-8.336).

\section{Survival Analysis}

In the univariate analysis (Kaplan-Meier, log rank test), the median OS was significantly associated with the factors of sex, primary tumour type, treatment of combined WBRT-GK, the presence of brain metastasectomy, KPS score, RPA class, BS-BM, DS-GPA, controlled primary tumour, and the absence of extracranial metastases $(p<0.05)$. The clinical characteristics and results of the univariate analysis of the OS of patients with BM are presented in Table II. The median OSs were 4 and 27 months among men and women, respectively $(p=0.00)$. Specifically, the median OS was 5 months for lung cancer, 9 months for other cancers (urogenital, colorectal cancer, malignant melanoma), and $>9$ months for breast cancer $(p=0.04)$. For the lung cancer subtypes, the median OS was 4 months for squamous cell carcinoma (SCC), and 6 months for both adenocarcinoma (AC) and small cell lung cancer (SCLC) $(p=0.00)$. The median OSs of the WBRT, GK, and combined WBRT-GK treatments were 6,4 , and 15 months, respectively $(p=0.04)$ (Figure 1). In our study, 24 of the 33 patients who underwent surgery, and 49 of the 58 patients who did not undergo surgery died. The median OS was 4 months and 14 months for the patients who did not undergo surgery and for those who underwent surgery, respectively $(p=0.01)$. Moreover, the median OSs were 9 and 4 months for patients with a KPS of $\geq 70$ and lower KPSs, respectively $(p=0.01)$. The median OS was significantly associated with the controlled primary tumour and the absence of extracranial metastases $(p<0.05)$. For the absence and the presence of primary tumour control, the median OSs were 4 months and 26 months, respectively $(p=0.00)$. The median OS was 15 months in patients without extracranial metastases, whereas it was 5 months in patients with extracranial metastases $(p=0.00)$. All prognostic index scores (RPA class, BS-BM, and DS-GPA) were closely related to the prognoses in our study from the univariate analysis (Kaplan-Meier, log rank test) (Figure 2). The median OSs were 20, 8, and 4 months for patients with RPA Class 1, RPA Class 2 , and RPA Class 3 , respectively $(\mathrm{p}=0.00)$. Further, the median OSs were 4, 6, 12, and 26 months for patients with BS-BM 0 , BS-BM 1, BS-BM 2, and BS-BM 3, respectively $(p=0.00)$. Meanwhile, for patients with DS-GPA 0-1 and DS-GPA 1.52.5 , the median OS was 6 months, whereas the median OS was $\geq 15$ months for patients with DS-GPA 3 and DS-GPA $3.5-4(p=0.00)$.

In the univariate analysis, we also found that the BMs $\geq 5$, total tumour volume $>4 \mathrm{~cm}^{3}$, male sex, absence of brain metastasectomy, and the KPS $\geq 70$ significantly correlated with the OS for the combined WBRT-GK treatment according 
to treatment modality. However, the total tumour volume $\leq 4$ $\mathrm{cm}^{3}$ significantly correlated with the OS for the GK treatment from the univariate analysis $(p<0.05)$. The results of the univariate analysis (Kaplan-Meier, log rank test) of the OS of the prognostic factors to the treatment modalities are presented in Table III. The OSs were different for different treatments among men. Accordingly, the median OSs were 4, 3 , and 9 months for the WBRT, GK, and the combined WBRT$\mathrm{GK}$, respectively $(\mathrm{p}=0.02)$. The median OS was not statistically significant according to the treatment modalities among women ( $p>0.05)$. In the absence of a brain metastasectomy, the median OS was 4 months for both the WBRT and GK

Table II: Clinical Characteristics and Results of the Univariate Analysis (Kaplan-Meier, Log Rank Test) of OS of Patients with Brain Metastasis

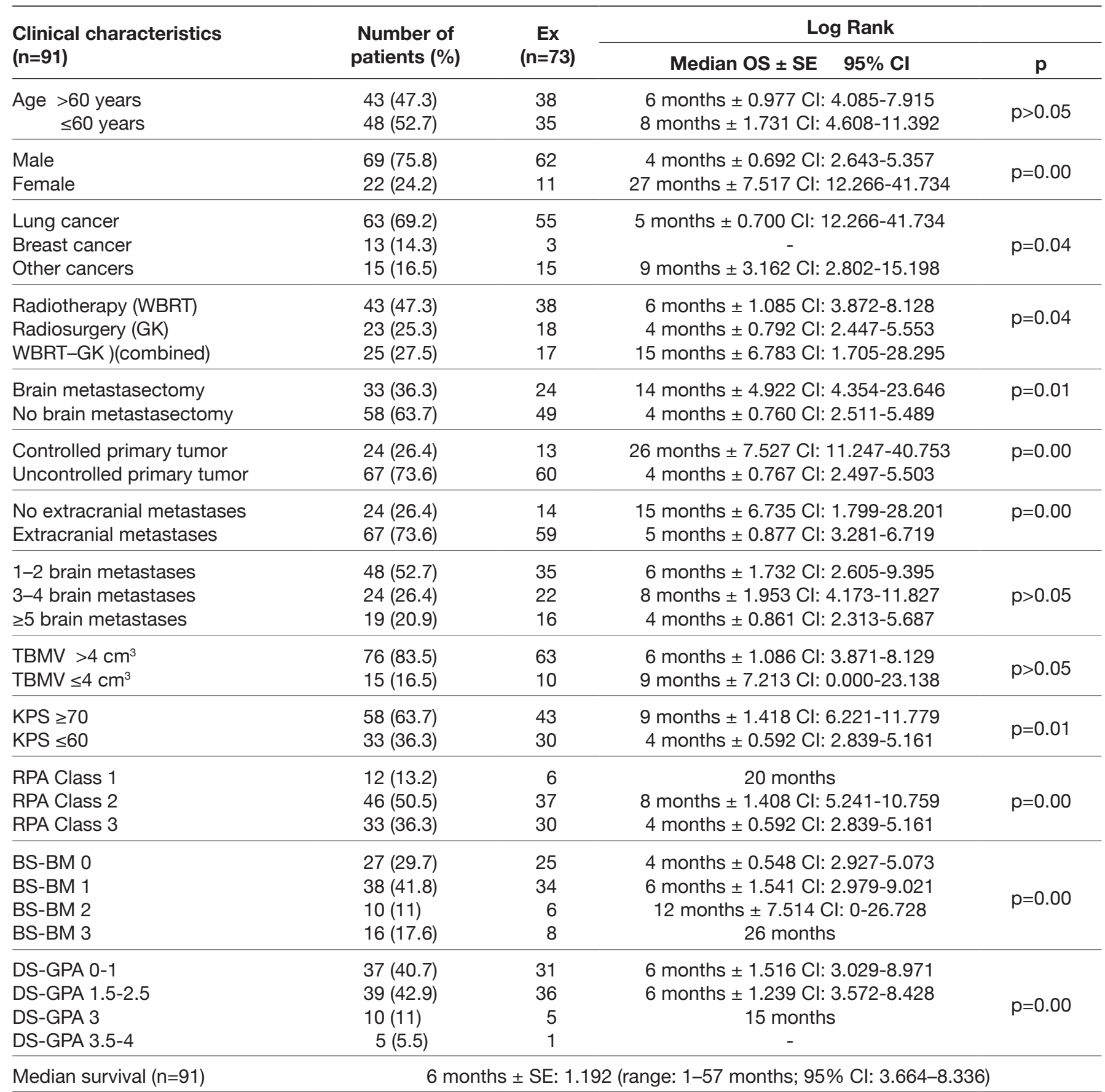

WBRT: Whole brain radiotherapy, GK: Gamma knife, KPS: Karnofsky performance status, RPA: Recursive partitioning analysis, BS-BM: Basic score for brain metastases, DS-GPA: Diseases specific-graded prognostic assessment, OS: Overall survival, TBMV: Total brain metastases volume, SE: Standard error, Cl: Confidence interval. 
treatments, and was 9 months for the combined WBRT-GK treatment $(p=0.00)$. According to the different treatments, the median OSs were not statistically significant for patients with a brain metastasectomy ( $p>0.05)$. Among the patients with oligo BMs, the median OS was not statistically significant according to the treatment modalities ( $p>0.05$ ). For those with 5 or more BMs, the median OS was 4 months for the WBRT and 1 month for the GK treatment, respectively, while for the combined WBRT-GK treatment, the OS was $>4$ months according to the different treatment modalities $(p=0.00)$. Moreover, for patients with $\mathrm{BM}$ volumes of $>4 \mathrm{~cm}^{3}$, the median OSs were 6,4 , and 10 months for the WBRT, GK, and combined WBRTGK treatments, respectively $(p=0.01)$. For patients with BM volumes of $\leq 4 \mathrm{~cm}^{3}$, the median OS was 1 month for the WBRT and 18 months for the combined WBRT-GK treatment, and $>18$ months for the GK treatment according to the different treatment modalities in the univariate analysis $(p=0.00)$. The median OSs were 7, 6, and 18 months for the WBRT, GK, and WBRT-GK treatments for patients with a KPS of $\geq 70$, respectively $(p=0.052)$. The median OS for patients with lower

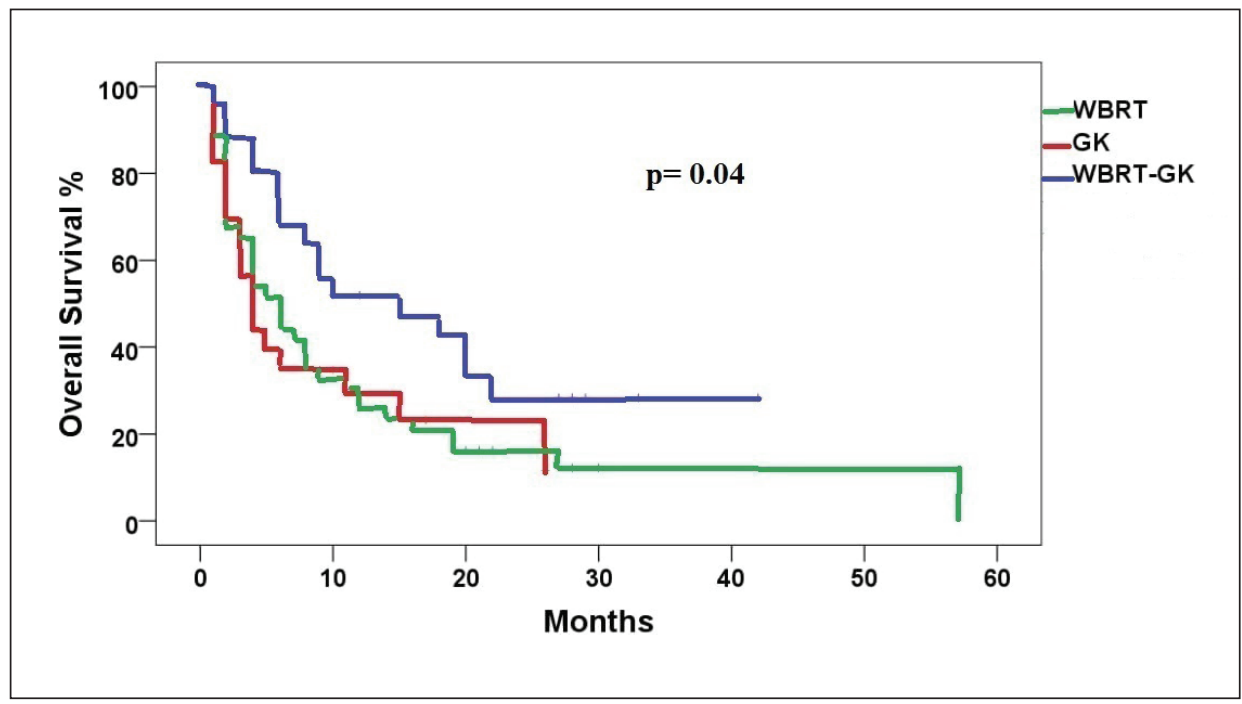

Figure 1: Kaplan-Meier analysis of the overall survival of patients with brain metastases according to treatment strategies.

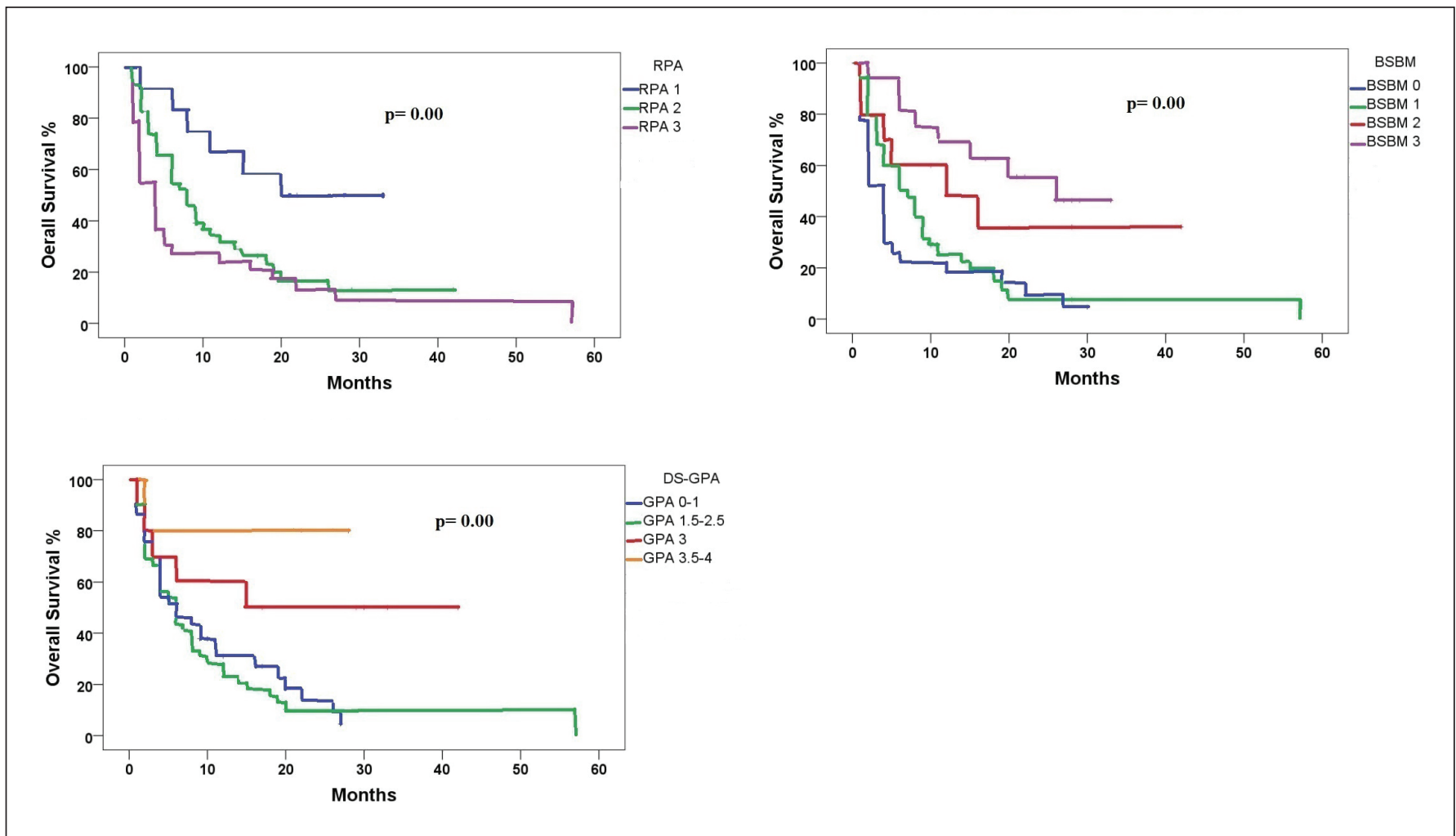

Figure 2: Kaplan-Meier analysis of the overall survival of patients with brain metastases according to the class of prognostic scores. 


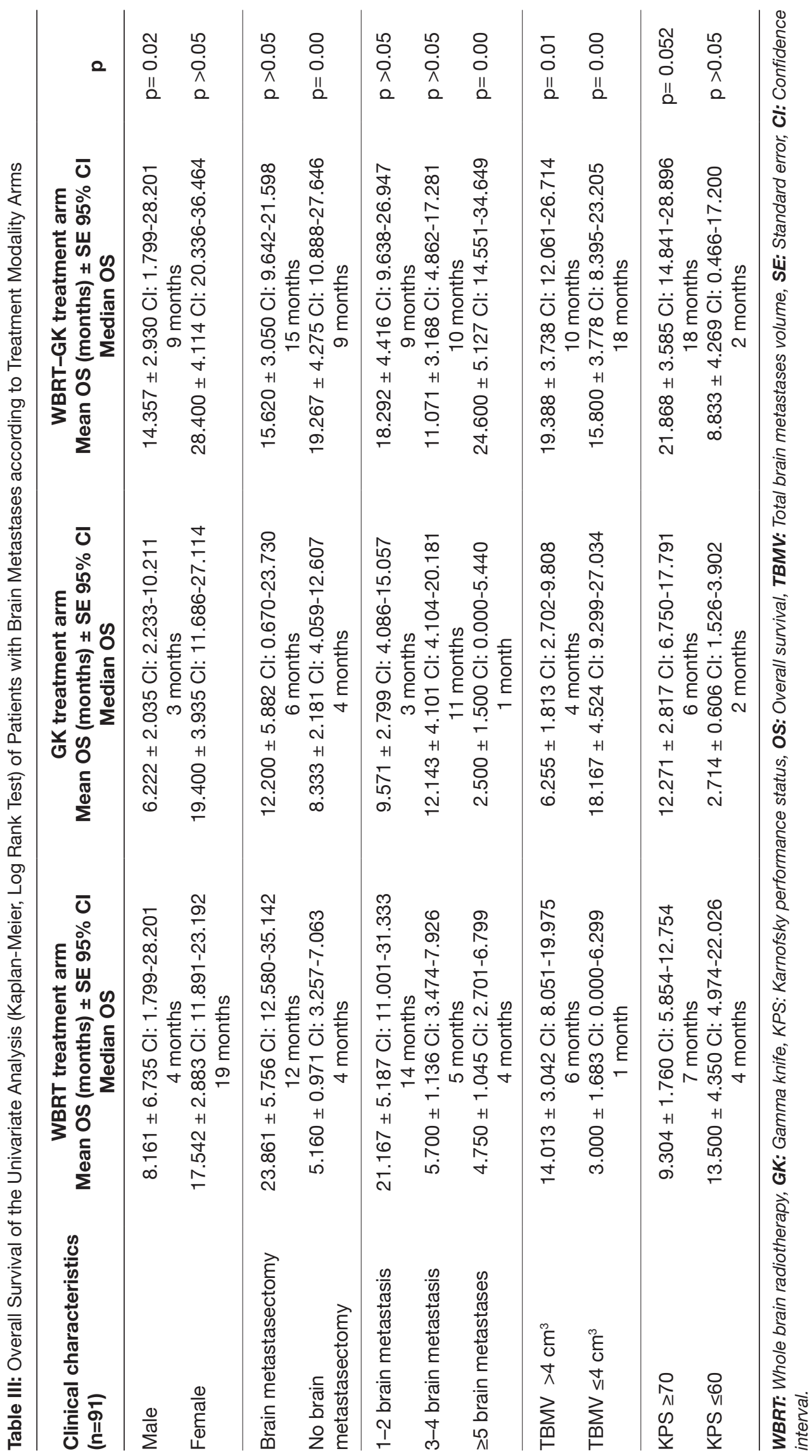


Saginc H. et al: Brain Metastases

Table IV: Results of the Multivariate Analysis (Cox-Regression Test) of Overall Survival of the Prognostic Factors and Prognostic Indexes

Clinical characteristics $(\mathrm{n}=91)$

Prognostic factors

Male vs.Female

Lung cancer

Breast cancer

Other cancers

Radiotherapy (WBRT)

Radiosurgery (GK)

WBRT-GK )(combined)

Brain metastasectomy vs. No brain metastasectomy

Controlled primary tumor vs. Uncontrolled primary tumor

No extracranial metastases vs. Extracranial metastases

KPS $\geq 70$ vs. KPS $\leq 60$

\section{Prognostic indexes}

RPA Class 1-2 vs. RPA Class 3

BS-BM 0-1 vs. BS-BM 2-3

DS-GPA $0-2.5$ vs. 3-4

\section{Cox Regression}

p

HR

$95 \% \mathrm{Cl}$

$\begin{array}{lll}p=0.030 & \text { HR: } 0.390 & \text { Cl: } 1.166-0.913 \\ p=0.499 & & \\ p=0.293 & \text { HR: } 0.449 & \text { Cl: } 0.101-1.997 \\ p=0.832 & \text { HR: } 1.078 & \text { Cl: } 0.539-2.155 \\ p=0.025 & & \\ p=0.891 & \text { HR: } 1.045 & \text { Cl: } 0.559-1.954 \\ p=0.015 & \text { HR: } 0.445 & \text { Cl: } 0.232-0.853 \\ p=0.047 & \text { HR: } 1.766 & \text { Cl: } 1.008-3.093 \\ p=0.007 & \text { HR: } 3.123 & \text { Cl: } 1.366-7.136 \\ p=0.244 & \text { HR: } 1.612 & \text { Cl: } 0.722-3.599 \\ p=0.591 & \text { HR: } 1.159 & \text { Cl: } 0.676-1.986\end{array}$

WBRT: Whole brain radiotherapy, GK: Gamma knife, KPS: Karnofsky performance status, RPA: Recursive partitioning analysis, BS-BM: Basic score for brain metastases, DS-GPA: Diseases specific-graded prognostic assessment, OS: Overall survival, SE: Standard error, CI: Confidence interval, HR: hazard ratio.

KPS scores was not statistically significant according to the different treatments $(p>0.05)$.

The multivariate analysis demonstrated that the female sex $(p=0.030)$, controlled primary tumour $(p=0.007)$, treatment with WBRT-GK $(p=0.001)$, and brain metastasectomy $(p=0.047)$ significantly correlated with the OS. The results of the multivariate analysis (the Cox regression model) of the OS of the prognostic factors are presented in Table IV. In the multivariate analysis, the controlled primary tumour significantly correlated with the OS, whereas, the absence of extracranial metastases showed negligible correlation with the OS. In the multivariate analysis, the BS-BM $(p=0.022)$ was closely related to the overall survival according to the class of prognostic index scores in our study. Additionally, in multivariate analysis, the BS-BM better predicted the survival of patients with BMs regarding the prognostic index scores. We found that the BS-BM was an appropriate prognostic score index in both the multivariate and univariate analyses. However, the RPA class and DS-GPA were not statistically significant in the multivariate analysis. The results of the multivariate analysis of the OSs according to the class of prognostic scores are presented in Table IV.

\section{DISCUSSION}

In our study, the median general survival among men and women was 4 and 27 months, respectively $(p=0.00)$. Harris et al. reported that the general survival of GK radiosurgery did not differ with respect to sex and previous WBRT applications (15). Similar to our study, many studies have shown better survival among women than men $(2,13,26)$. Although the survival of men with brain metastasis is poor, our study findings confirm that the survival rate of men improves following WBRT-GK treatment. Considering these findings, this combined therapy may be preferred for men.

In our study, including 63 patients with lung cancer, for the lung cancer subtypes, the median OS was 4 months for SCC, and 6 months for AC and SCLC $(p=0.00)$. In addition, the median survival of lung cancer was 4 months for both the WBRT and GK treatments, while that for the WBRT-GK treatment was 8 months. In our study, patients with BMs from AC and SCLC generally had a better survival rate. In addition, for patients with BMs from lung cancer, the combined WBRTGK treatment had a better OS. In a study by Kuremsky et al. which included 271 patients, the median survival was 10.2, 5.9, and 5.3 months for patients with BMs from ACA, SCLC, and SCC, respectively. For the SCLC and SCC histology types predicted at an earlier time for salvage WBRT, the OS worsened. The authors reported that the histological subtype may represent a new variable between the current prognostic indexes. Compared with SCC, AC has had a better success in recent years due to the success of systemic treatment, as reported in our study (20). In cases of wild-type EGFR and ALK NSCLC, there are few effective systemic options; therefore, WBRT may have a more prominent role. Despite the current trend of preferring SRS alone, the important role of WBRT should be carefully considered, particularly for patients with BMs from NSCLC who have a favourable prognosis (17).

Similar to our study, the median OS was the highest among patients with breast cancer compared with that of patients with lung cancer and other cancer types $(13,15,26)$. In another study, no difference was reported between the OSs of patients 
with breast and lung cancers (9). In the study by Miller which included 547 patients with BMs from breast cancer, the median survival was not enhanced by hormone therapy in the cases with positive hormone receptors (15.3-13.9 months, $p=0.21$ ). The use of both HER2 antibody drugs (17.9 vs 15.1 months; $\mathrm{p}=0.04)$ and HER2/EGFR TKIs (21.1-15.4 months, $\mathrm{p}=0.03$ ) increased the survival rate. Molecular subtypes are prognostic factors for survival and determinant factors for the response to radiotherapy (25). Likewise, the agents used were believed to improve the survival of patients with breast cancer in our study as well, for which the highest survival was observed.

In the study by Bowden, the presence of multiple BMs is a negative predictor of a worse prognosis (6). In other studies, the number of metastatic lesions of $1-2$ vs $3-4$ vs. $\geq 5$ did not approach significance. The importance of the number of BMs vs. the total tumour volume in predicting the OS remains debatable $(9,25,40)$. In our multivariate analyses, the total tumour volume and number of BMs were not statistically significantly associated with OS. In the univariate analyses, we found that the total tumour volume was statistically significant only according to the different treatment modalities. However, the median OS was highest for the combined WBRT-GK treatment for patients with BM volumes of $>4 \mathrm{~cm}^{3}$ and for the GK treatment of patients with BM volumes of $\leq 4 \mathrm{~cm}^{3}$. Many studies have shown that the tumour volume was statistically significantly correlated with the OS $(4,5,21)$. Thus, the total tumour volume should be examined more closely in future studies. In our study, the median survival rate of patients with $\geq 5$ metastases was lower than that of those with oligo metastases $(p>0.05)$. Moreover, the median survival was the highest for patients with $\geq 5 \mathrm{BMs}$ for the combined WBRT-GK treatment $(p=0.00)$. For patients with $\geq 5$ BMs, GK salvage therapy may be applied with WBRT (37). Considering the survival benefit of the combination of WBRT and SRS, hippocampus sparing-WBRT studies are required to reduce the cognitive effects of WBRT (14).

In our study, the median survival of patients who underwent a brain metastasectomy was better than those who did not undergo a brain metastasectomy, which was statistically significant. The combined WBRT-GK treatment improved the OS of those patients who did not undergo brain metastasectomy. The combined WBRT-GK treatment was not related with the median OS in patients undergoing a brain metastasectomy. In two trials, $(33,43)$ a survival benefit was reported for patients undergoing combined brain metastasectomy and WBRT.

Similar to other studies, we demonstrated that the OS is better for patients with $\mathrm{KPS} \geq 70$ from the univariate analysis $(31,32)$. Xiaoyu et al. showed that the OS increased for patients with a favourable KPS score from univariate and multivariate analyses (42).

In our study, the OS increased for patients with a favourable KPS, better control of extracranial disease, and controlled primary tumour, as reported by Gao et al. (10). Our series also matches with previous reports which have demonstrated an improved median OS with better control of extracranial disease $(35,42)$. The OS was also better in the study of 294 cases with controlled primary tumours, similar to our study (9). However, another study reported that the OS was improved in patients with controlled primary tumours and a previous metastasectomy (15).

RPA Class 1 (Class 1, median survival 20 months) was different from the original data because the number of patients in RPA Class 1 in this study was small. In the first RTOG study by Gaspar et al. (12), the median OS of patients with RPA Class 1 was 7.1 months. In another study including 445 cases, also by Gaspar et al. (11) the median OS was 6.2 months for RPA 1 and 3.8 months for RPA 2. In a study of 528 cases by Nieder, the RPA class received similar results, but the number of patients in RPA Class 1 was very small (28). In the study of 110 patients by Lorenzoni et al., the median OS was 27.6 months for RPA Class 1 and 10.7 months for RPA Class 2, similar to our study (23).

In our multivariate analysis, the BS-BM $(p=0.022)$ better predicted the survival of BM. Lorenzoni reported that the median OS was undefined for BS-BM 3 (55\% at 32 months) and was 13.1 months for BS-BM 2. However, the median OS was 26 months for BS-BM 3 and 12 months for BS-BM 2 in our study (23). Similar to our multivariate analysis, Villa et al. reported that the median OS improved for the BS-BM $(p<0.001)$. In this prospective study, the prognostic indexes of the RPA, BS-BM, and GPA scores were prognostically relevant in BM patients, similar to those in our univariate analysis (44). In another retrospective study of 335 patients by Xiaoyu et al., the RPA, BS-BM, and GPA scores were statistically significant for the OS, similar to our study (42). In the study of 1,960 cases with BM by Sperduto, the median OS was 6.9 and 11.0 months in cases with GPA 3 and GPA 3.5-4.0, respectively. In our study with a small number of patients, the median OS was better for GPA (15 months for GPA 3; >15 months for GPA 3.5-4.0) (18).

\section{CONCLUSION}

From the multivariate analysis, we found that female sex, control of primary tumours, combined WBRT-GK treatment, and brain metastasectomy were statistically significant for the OS of patients with BMs. In our study, all the published prognostic indexes assessed could predict patient prognosis, demonstrating the reliability and clinical relevance of these scores. However, the BS-BM better predicted the survival of patients with BMs according to the prognostic index scores from the multivariate analysis. Our data suggest that the BS$\mathrm{BM}$ is the most appropriate prognostic index.

\section{- ACKNOWLEDGEMENTS}

We thank the medical staff of the Department of Neurosurgery and Department of Radiation Oncology, School of Medicine, University of Pamukkale, for their cooperation in relation to this paper. We also thank medical statistician Hande Senol of the Department of Medical Statistics, School of Medicine, University of Pamukkale. 


\section{REFERENCES}

1. Andrews DW, Scott CB, Sperduto PW: Whole brain radiation therapy with or without stereotactic radiosurgery boost for patients with one to three brain metastases: Phase III results of the RTOG 9508 randomised trial. Lancet 363:1665-1672, 2004

2. Aoyama $\mathrm{H}$, Shirato $\mathrm{H}$, Tago M: Stereotactic radiosurgery plus whole-brain radiation therapy vs stereotactic radiosurgery alone for treatment of brain metastases: A randomized controlled trial. JAMA 295:2483-2491, 2006

3. Bhatnagar AK, Flickinger JC, Kondziolka D: Stereotactic radio-surgery for four or more intracranial metastases. Int $\mathrm{J}$ Radiat Oncol Biol Phys 64:898-903, 2006

4. Bhatnagar AK, Flickinger JC, Kondziolka D: Stereotactic radiosurgery for four or more intracranial metastases. Int $\mathrm{J}$ Radiat Oncol Biol Phys 64:898-903, 2006

5. Baschnagel AM, Meyer KD, Peter YC: Tumor volume as a predictor of survival and local control in patients with brain metastases treated with Gamma Knife surgery Clinical article. Journal of Neurosurgery 119:1139-1144, 2013

6. Bowden G, Kano H, Caparosa E: Gamma Knife radiosurgery for the management of cerebral metastases from non-small cell lung cancer. J Neurosurg 122:766-772, 2015

7. Bradley KA, Mehta MP: Management of brain metastases. Semin Oncol 31:693-701, 2004

8. Chang EL, Wefel JS, Hess KR: Neurocognition in patients with brain metastases treated withradiosurgery or radiosurgery plus whole-brain irradiation: A randomised controlled trial. Lancet Oncol 10:1037-1044, 2009

9. Ebner DK, Gorovets D, Rava P: Patients with long-term control of systemic disease are a favorable prognostic group for treatment of brain metastases with stereotactic radiosurgery alone. World Neurosurg 98:266-272, 2017

10. Gao HX, Huang SG, Du JF: Comparison of prognostic indices in NSCLC patients with brain metastases after radiosurgery. International Journal of Biological Sciences 14(14):20652072, 2018

11. Gaspar LE, Scott C, Murray K: Validation of the RTOG recursive partitioning analysis (RPA) classification for brain metastases. International Journal of Radiation Oncology* Biology* Physics 47:1001-1006, 2000

12. Gaspar L, Scott C, Rotman M: Recursive partitioning analysis (RPA) of prognostic factors in three Radiation Therapy Oncology Group (RTOG) brain metastases trials. Int J Radiat Oncol Biol Phys 37:745-751,1997

13. Greenspoon JN, Ellis PM, Pond G: Comparative survival in patients with brain metastases from non-small-cell lung cancer treated before and after implementation of radiosurgery. Curr Oncol 24:146-151, 2017

14. Gondi V, Pugh SL, Tome WA: Preservation of memory with conformal avoidance of the hippocampal neural stem cell compartment during whole-brain radiotherapy for brain metastases (RTOG 0933): A phase II multi-institutional trial. Journal of Clinical Oncology 32:3810-3816, 2014

15. Harris $\mathrm{BH}$, Corbett MR, Mascarenhas $\mathrm{H}$ : A single-institution analysis of 126 patients treated with stereotactic radiosurgery for brain metastases. Front Oncol 7:90, 2017
16. Kaplan EL, Meier P: Non parametric estimation from incomplete observations. J Am Stat Assoc 53:457-481, 1958

17. Kevin S, Loeffler JS: The changing landscape of whole-brain radiation therapy invited commentary. JAMA Oncol 1:464465, 2015

18. Kocher M, Wittig A, Piroth MD: Stereotactic radiosurgery for treatment of brain metastases. A report of the DEGRO Working Group on Stereotactic Radiotherapy. Strahlenther Onkol 190:521-532, 2014

19. Kocher M, Soffietti R, Abacioglu U: Adjuvant whole brain radiotherapy versus observation after radiosurgery or surgical resection of one to three cerebral metastases: Results of the EORTC 22952-26001 study. J Clin Oncol 29:134-141, 2011

20. Kuremsky JG, Urbanic JJ, Petty WJ: Tumor histology predicts patterns of failure and survival in patients with brain metas-tases from lung cancer treated with Gamma Knife Radiosurgery. Neurosurgery 73:641-647, 2013

21. Likhacheva A, Pinnix CC, Parikh NR: Predictors of survival in contemporary practice after initial radiosurgery for brain metastases. Int J Radiat Oncol Biol Phys 85:656-661, 2012

22. Lin NU, Bellon JR, Winer EP: CNS metastases in breast cancer. J Clin Oncol 22:3608-3617, 2004

23. Lorenzoni J, Devriendt D, Massager N: Radiosurgery for treatment of brain metastases: Estimation of patient eligibility using three stratification systems. Int J Radiat Oncol Biol Phys 60:218-224, 2004

24. Mehta MP, Tsao MN, Whelan TJ: The American Society for Therapeutic Radiology and Oncology (ASTRO) evidencebased review of the role of radiosurgery for brain metastases. Int J Radiat Oncol Biol Phys 63:37-46, 2005

25. Miller JA, Kotecha R, Ahluwalia MS: Overall survival and the response to radiotherapy among molecular subtypes of breast cancer brain metastases treated with targeted therapies. Cancer 123:2283-2293, 2017

26. Mohammadi AM, Recinos PB, Barnett GH: Role of Gamma Knife surgery in patients with 5 or more brain metastases. J Neurosurg 117 Suppl:5-12, 2012

27. Nayak L, Lee EQ, Wen PY: Epidemiology of brain metastases. Curr Oncol Rep 14:48-54, 2012

28. Nieder C, Nestle U, Motaref B: Prognostic factors in brain metastases: Should patients be selected for aggressive treatment according to recursive partitioning analysis (RPA) classes? Int J Radiat Oncol Biol Phys 46:297-302, 2000

29. Nussbaum ES, Djalilian HR, Cho KH: Brain metastases. Histology, multiplicity, surgery and survival. Cancer 78:17811788, 1996

30. Owen S, Souhami L: The management of brain metastases in non-small cell lung cancer. Front Oncol 4:248, 2014

31. Page BR, Wang EC, White L: Gamma Knife radiosurgery for brain metastases from gastrointestinal primary. J Med Imaging Radiat Oncol 61:522-527, 2017

32. Patchell RA: The management of brain metastases. Cancer Treat Rev 29:533-540,2003

33. Patchell RA, Tibbs PA, Walsh JW, Dempsey RJ: A randomized trial of surgery in the treatment of single metastases to the brain. N Engl J Med 322(8):494-500, 1990 
34. Pirzkall A, Debus J, Lohr F: Radiosurgery alone or in combination with whole brain radiotherapy for brain metastases. J Clin Oncol 16:3563-3569, 1998

35. Rades D, Janssen S, Bajrovic A: A matched-pair analysis comparing whole brain radiotherapy with and without a stereotactic boost for intracerebral control and overall survival in patients with one to three cerebral metastases. Radiat Oncol 12:69, 2017

36. Rades D, Dziggel SJL, Blanck O: A matched-pair study comparing whole brain irradiation alone to radiosurgery or fractionated stereotactic radiotherapy alone in patients irradiated for up to three brain metastases. BMC Cancer 17:30, 2017

37. Sengöz M, Kabalay IA, Tezcanlı E, Peker S, Pamir N: Treatment of brainstem metastases with gamma-knife radiosurgery. J Neurooncol 113:33-38, 2013

38. Soon YY, Tham IW, Lim KH: Surgery or radiosurgery plus whole brain radiotherapy versus surgery or radiosurgery alone for brain metastases. Cochrane Database Syst Rev 3: CD009454, 2014

39. Sperduto PW, Berkey B, Gaspar LE: A new prognostic index and comparison to three other indices for patients with brain metastases: An analysis of 1,960 patients in the RTOG database. Int J Radiat Oncol Biol Phys 70:510-514, 2008
40. Sperduto PW, Chao ST, Sneed PK: Diagnosis-specific prognostic factors, indexes, and treatment outcomes for patients with newly diagnosed brain metastases: A multiinstitutional analysis of 4259 patients. Int $\mathrm{J}$ Radiat Oncol Biol Phys 77:655-661, 2010

41. Tsao MN, Llyod N, Wong RK: Whole brain radiotherapy for the treatment of newly diagnosed multiple brain metastases. Cochrane Database Syst Rev 18:CD003869, 2012

42. Xiaoyu J, Yingjie Z, Xiangye Y: Survival time following resection of intracranial metastases from NSCLC development and validation of a novel nomogram. BMC Cancer 17:774, 2017

43. Vecht CJ, Haaxma-Reiche $H$, Noordijk EM: Treatment of single brain metastasis: Radiotherapy alone or combined with neurosurgery? Ann Neurol 33(6):583-590, 1993

44. Villà S, Weber DC, Moretones C, Mañes A: Validation of the new graded prognostic assessment scale for brain metastases: A multicenter prospective study. Radiation Oncology 6:23, 2011

45. Yomo S, Hayashi M: The efficacy and limitations of stereotactic radiosurgery as a salvage treatment after failed whole brain radiotherapy for brain metastases. Journal of Neuro-Oncology 113:459-465, 2013 